\title{
On groups covered by locally nilpotent subgroups
}

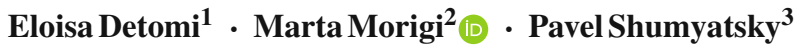

Received: 16 March 2016 / Accepted: 7 November 2016 / Published online: 15 November 2016

(C) Fondazione Annali di Matematica Pura ed Applicata and Springer-Verlag Berlin Heidelberg 2016

\begin{abstract}
Let $\mathcal{N}$ be the class of pronilpotent groups, or the class of locally nilpotent profinite groups, or the class of strongly locally nilpotent profinite groups. It is proved that a profinite group $G$ is finite-by- $\mathcal{N}$ if and only if $G$ is covered by countably many $\mathcal{N}$-subgroups. The commutator subgroup $G^{\prime}$ is finite-by- $\mathcal{N}$ if and only if the set of all commutators in $G$ is covered by countably many $\mathcal{N}$-subgroups. Here, a group is strongly locally nilpotent if it generates a locally nilpotent variety of groups. According to Zelmanov, a locally nilpotent group is strongly locally nilpotent if and only if it is $n$-Engel for some positive $n$.
\end{abstract}

Keywords Profinite groups $\cdot$ Coverings $\cdot$ Commutators

Mathematics Subject Classification $20 \mathrm{E} 18 \cdot 20 \mathrm{~F} 12 \cdot 20 \mathrm{~F} 14$

\section{Introduction}

A covering of a group $G$ is a family of subsets of $G$ whose union is the whole group $G$. When $G$ is covered by finitely many subgroups, it is natural to expect that some structural

This research was partially supported by Università di Padova (Progetto di Ricerca di Ateneo: "Invariable generation of groups"). The third author was also supported by FAPDF and CAPES.

$凶$ Marta Morigi

marta.morigi@unibo.it

Eloisa Detomi

detomi@math.unipd.it

Pavel Shumyatsky

pavel@unb.br

1 Dipartimento di Matematica, Università di Padova, Via Trieste 63, 35121 Padua, Italy

2 Dipartimento di Matematica, Università di Bologna, Piazza di Porta San Donato 5, 40126 Bologna, Italy

3 Department of Mathematics, University of Brasilia, Brasilia, DF 70910-900, Brazil 
information about $G$ can be deduced from the properties of the covering subgroups. In the literature, there are many results in this direction. If the set of all commutators $[x, y]$ in a group $G$ is contained in a union of finitely many subgroups, it is natural to ask whether the structure of the commutator subgroup $G^{\prime}$ depends on the properties of the covering subgroups. The questions become even more intriguing when the groups are profinite. In that case the interplay of algebraic and topological considerations adds a new quality to the obtained results. One important tool for dealing with the "covering" problems in profinite groups is the classical Baire's category theorem (cf [5, p. 200]): If a locally compact Hausdorff space is a union of countably many closed subsets, then at least one of the subsets has non-empty interior. It follows that if a profinite group is covered by countably many subgroups, then at least one of the subgroups is open (here, and throughout the article, by a subgroup of a profinite group, we mean a closed subgroup). Thus, in case of profinite groups, we can deal with problems on countable coverings rather than just finite ones. A survey of recent results of that kind can be found in [1].

In the present paper, we address questions closely related to the following results obtained in [9]: A profinite group $G$ is covered by countably many nilpotent subgroups if and only if $G$ is finite-by-nilpotent, and the set of commutators in a profinite group $G$ is covered by countably many nilpotent subgroups if and only if $G^{\prime}$ is finite-by-nilpotent.

Throughout, we use the same symbol to denote a group-theoretical property and the class of groups with that property. Following [7], whenever $X$ and $Y$ are properties of groups, or classes of groups, the expression " $G$ is $X$-by- $Y$ " means that the group $G$ is an extension of a group with property $X$ (resp. in $X$ ) by a group with $Y$ (resp. in $Y$ ).

Recall that a group $G$ is locally nilpotent if all finitely generated subgroups of $G$ are nilpotent. Following Shalev [8], we say that a group $G$ is strongly locally nilpotent if it belongs to a locally nilpotent variety of groups. This means that, for some function $f$ and for all positive integers $d$, every $d$-generated subgroup of $G$ is nilpotent of class at most $f(d)$.

The purpose of the present article is to obtain the following results.

Theorem 1.1 Let $\mathcal{N}$ be the class of pronilpotent groups, or the class of locally nilpotent profinite groups, or the class of strongly locally nilpotent profinite groups. For a profinite group $G$ the following conditions are equivalent:

1. The group $G$ is covered by countably many $\mathcal{N}$-subgroups.

2. The group $G$ is covered by finitely many $\mathcal{N}$-subgroups.

3. The group $G$ is finite-by- $\mathcal{N}$.

Theorem 1.2 Let $\mathcal{N}$ be the class of pronilpotent groups, or the class of locally nilpotent profinite groups, or the class of strongly locally nilpotent profinite groups. For a profinite group $G$ the following conditions are equivalent:

1. The set of all commutators in $G$ is covered by countably many $\mathcal{N}$-subgroups.

2. The set of all commutators in $G$ is covered by finitely many $\mathcal{N}$-subgroups.

3. The commutator subgroup $G^{\prime}$ is finite-by- $\mathcal{N}$.

Amazingly, no analogues of Theorem 1.2 for abstract groups are known even in the case of finite coverings. There are no results in the literature concerning the structure of abstract groups in which the commutators are covered by finitely many nilpotent, or locally nilpotent, subgroups. A characterization of (abstract) groups covered by finitely many nilpotent subgroups can be found in Tomkinson [12]. The results from [12] are not used in the present article. 
One noteworthy corollary of our results is that whenever the set of commutators in $G$ is covered by countably many $\mathcal{N}$-subgroups, the whole commutator subgroup $G^{\prime}$ admits a similar covering.

Corollary 1.1 Let $\mathcal{N}$ be the class of pronilpotent groups, or the class of locally nilpotent profinite groups, or the class of strongly locally nilpotent profinite groups. For a profinite group $G$ the following condition are equivalent:

1. The set of all commutators in $G$ is covered by countably many $\mathcal{N}$-subgroups.

2. The commutator subgroup $G^{\prime}$ is covered by countably many $\mathcal{N}$-subgroups.

A few words about pronilpotent, locally nilpotent and strongly locally nilpotent groups are in order. We recall that a pronilpotent group is an inverse limit of finite nilpotent groups; hence, it is a direct product of its Sylow subgroups. Notice also that a locally nilpotent profinite group is pronilpotent. An element $x \in G$ is called a (left) Engel element if for any $g \in G$ there exists $n=n(x, g) \geq 1$ such that $\left[g,{ }_{n} x\right]=1$, where the commutator $\left[g,{ }_{n} x\right]$ is defined recursively by the rule

$$
[g, n]=[[g, n-1 x], x]
$$

starting with $[g, 0 x]=g$ and $\left[g,{ }_{1} x\right]=[g, x]=g^{-1} x^{-1} g x$. If $n$ can be chosen independently of $g$, then $x$ is a (left) $n$-Engel element. A group $G$ is called Engel if all elements of $G$ are Engel and $G$ is called $n$-Engel if all elements of $G$ are $n$-Engel. It is clear that each locally nilpotent group is Engel. In [14], Wilson and Zelmanov proved that for profinite groups the converse holds: An Engel profinite group is necessarily locally nilpotent.

A variety of groups is a class of groups defined by equations. More precisely, if $W$ is a set of words, the class of all groups satisfying the laws $W \equiv 1$ is called the variety determined by $W$. By a well-known theorem of Birkhoff [7, 2.3.5], varieties are precisely classes of groups closed with respect to taking subgroups, quotients, and Cartesian products of their members. Some interesting varieties of groups have been discovered in the context of the restricted Burnside problem solved in the affirmative by Zelmanov [16, 17]. In [15], Zelmanov remarked that the solution of the restricted Burnside problem implies that the class of locally nilpotent $n$-Engel groups is a variety (see also Wilson [13]). Thus, it follows that a locally nilpotent group is strongly locally nilpotent if and only if it is $n$-Engel for some $n$.

Throughout the article, by a subgroup of a profinite group, we mean a closed subgroup, in particular by "subgroup generated by a set" or "product of a family of normal subgroup" we mean their topological closure. Moreover, the letter $\mathcal{N}$ will denote one of the following classes: the class of pronilpotent groups, the class of locally nilpotent profinite groups, or the class of strongly locally nilpotent profinite groups. In addition, we use the expression " $\{a, b, \ldots\}$-bounded" to abbreviate "bounded from above in terms of $a, b, \ldots$ only".

\section{Preliminary results}

In the present section, we will develop some useful tools that will be later used for the proofs of our main results. We start with an elementary lemma on coprime automorphisms. If $\alpha$ is an automorphism of a group $G$, as usual $[G, \alpha]$ denotes the subgroup generated by all elements of the form $x^{-1} x^{\alpha}$, where $x \in G$. It is well known that $[G, \alpha]$ is a normal subgroup of $G$. We denote by $C_{G}(\alpha)$ the fixed point subgroup (centralizer) of $\alpha$ in $G$.

Lemma 2.1 Let $G$ be a finite group and let $\alpha$ be an automorphism of $G$ such that $G$ and $\alpha$ have coprime orders. If $C_{G}(\alpha)$ has finite index $k$ in $G$, then the order of $[G, \alpha]$ is $k$-bounded. 
Proof By $[2,24.5]$ we have $[G, \alpha]=[G, \alpha, \alpha]$. So we may replace $G$ with $[G, \alpha]$ and assume that $G=[G, \alpha]$. Let $K$ be a normal subgroup of $G$ which is maximal with respect to the condition that $K \leq C_{G}(\alpha)$. Of course the index of $K$ in $G$ is $k$-bounded. For each $h \in K$ and $x \in G$, we have $h^{x} \in K \leq C_{G}(\alpha)$. Therefore, $h^{x}=\left(h^{x}\right)^{\alpha}=h^{x^{\alpha}}$. We see that every $h \in K$ commutes with $x^{-1} x^{\alpha}$ for each $x \in G$. Since $G=[G, \alpha]$, it follows that $K$ is contained in the center of $G$. By Schur's theorem [7, 10.1.4], the order of $G^{\prime}$ is $k$-bounded and, by passing to the quotient $G / G^{\prime}$, we may assume that $G$ is abelian. In that case $G=[G, \alpha] \times C_{G}(\alpha)$, so now $|[G, \alpha]| \leq k$ and the result follows.

By an automorphism of a profinite group, we always mean a continuous automorphism. An automorphism $\alpha$ of a profinite group $G$ is coprime if $\alpha$ has finite order while $G$ is an inverse limit of finite groups whose orders are relatively prime to the order of $\alpha$. The next corollary can be deduced from Lemma 2.1 using the standard inverse limit argument.

Corollary 2.1 Let $G$ be a profinite group admitting a coprime automorphism $\alpha$. If the centralizer of $\alpha$ in $G$ is open, then $[G, \alpha]$ is finite.

As mentioned in the introduction, strongly locally nilpotent groups are precisely locally nilpotent groups that are $n$-Engel for some positive integer $n$. Obviously, a profinite group having a dense strongly locally nilpotent subgroup is strongly locally nilpotent, too. We therefore can establish some basic facts on abstract strongly locally nilpotent groups and then use those facts in the profinite context.

We say that an automorphism $\alpha$ of a group $G$ is $s$-Engel if it is a left $s$-Engel element in the semidirect product $G\langle\alpha\rangle$.

Lemma 2.2 Let $G$ be an n-Engel locally nilpotent group and let a be an s-Engel automorphism of $G$. Then for every $x, y \in G$ the subgroup $\langle x, y, a\rangle$ is nilpotent of $(s, n)$-bounded class.

Proof Set $H=\langle x, y\rangle$. By [3, Corollary 2] the subgroup $\left\langle H^{\langle a\rangle}\right\rangle$ is finitely generated and, more specifically, the minimal number $r$ of generators of $\left\langle H^{\langle a\rangle}\right\rangle$ is $s$-bounded. According to Zelmanov [15], the nilpotency class of $\left\langle H^{\langle a\rangle}\right\rangle$ is $(s, n)$-bounded. Hence, the derived length of $\langle x, y, a\rangle$ is $(s, n)$-bounded. It is clear that both $x$ and $y$ are $(n+1)$-Engel in $\langle x, y, a\rangle$ and so the subgroup $\langle x, y, a\rangle$ is soluble with bounded derived length and is generated by three elements that are Engel of bounded degree. By [10, Lemma 4.1] $\langle x, y, a\rangle$ is nilpotent of $(s, n)$-bounded class, as required.

Lemma 2.3 Let $G$ be a strongly locally nilpotent group and let a be an s-Engel automorphism of $G$. Then $G\langle a\rangle$ is strongly locally nilpotent. More precisely, if $G$ is $n$-Engel, then $G\langle a\rangle$ is $m$-Engel for some $(s, n)$-bounded integer $m$.

Proof Choose arbitrarily two elements $g_{1}$ and $g_{2}$ in $G\langle a\rangle$. Write $g_{1}=x a_{1}$ and $g_{2}=y a_{2}$, where $x, y \in G$ and $a_{1}, a_{2} \in\langle a\rangle$. It is clear that $g_{1}, g_{2} \in\langle x, y, a\rangle$. Hence, by Lemma 2.2, the subgroup $\left\langle g_{1}, g_{2}\right\rangle$ is nilpotent of $(s, n)$-bounded class, say $m$. Therefore, $G\langle a\rangle$ is $m$-Engel.

Lemma 2.4 In any group a product of finitely many strongly locally nilpotent normal subgroups is again strongly locally nilpotent.

Proof By standard arguments, it is sufficient to prove the lemma for a product of two normal subgroups. Thus, assume that $N_{1}$ and $N_{2}$ are normal strongly locally nilpotent subgroups in a group $G$. Suppose that $N_{i}$ is $m_{i}$-Engel for some $m_{i}$, where $i=1,2$. Without loss of generality, we assume that $m_{1} \geq m_{2}$. We need to show that there exists an $m_{1}$-bounded number $t$ such 
that an arbitrary element $x \in N_{1} N_{2}$ is $t$-Engel in $N_{1} N_{2}$. Let $N_{0}=N_{1} \cap N_{2}$. Clearly, the quotient $N_{1} N_{2} / N_{0}$ is $m_{1}$-Engel. Therefore, it is sufficient to show that the automorphism induced by $x$ on $N_{0}$ is $s$-Engel for some $m_{1}$-bounded number $s$. Write $x=x_{1} x_{2}$, where $x_{1} \in N_{1}$ and $x_{2} \in N_{2}$. Since $\left[N_{2}, x_{1}\right] \leq N_{0}$, it is clear that the automorphism induced by $x_{1}$ on $N_{2}$ is $\left(m_{1}+1\right)$-Engel. Now choose $y \in N_{0}$. We will be done, once we show that $\langle x, y\rangle$ has $m_{1}$-bounded nilpotency class. Observe that $\langle x, y\rangle \leq\left\langle x_{1}, x_{2}, y\right\rangle$. Both $x_{2}, y$ are contained in $N_{2}$. Hence, by Lemma 2.2, the subgroup $\left\langle x_{1}, x_{2}, y\right\rangle$ has bounded nilpotency class.

We will also need a slightly more general version of the previous lemma. Recall that the $\mathcal{N}$-residual $G^{\mathcal{N}}$ of an abstract group $G$ is the intersection of all normal subgroups $D$ such that $G / D$ is in $\mathcal{N}$. In general, $G / G^{\mathcal{N}}$ might fail to be a $\mathcal{N}$-group, but if $G$ is finite-by- $\mathcal{N}$, then $G / G^{\mathcal{N}}$ is in $\mathcal{N}$. This follows from the fact that a product of finitely many normal $\mathcal{N}$ subgroups is in $\mathcal{N}$, which is well known for the class of pronilpotent and locally nilpotent groups, and is Lemma 2.4 for the class of strongly locally nilpotent groups.

Lemma 2.5 In any group a product of finitely many finite-by- $\mathcal{N}$ normal subgroups is again finite-by-N.

Proof By standard arguments, it is sufficient to prove the lemma for a product of two normal $\mathcal{N}$-subgroups $N_{1}$ and $N_{2}$ of a group $G$. Let $N_{i}^{\mathcal{N}}$ be the $\mathcal{N}$-residual of $N_{i}$, for $i=1,2$. As each $N_{i}^{\mathcal{N}}$ is characteristic in $N_{i}$, we have that $N_{1}^{\mathcal{N}} N_{2}^{\mathcal{N}}$ is a finite normal subgroup of $N_{1} N_{2}$ and the quotient $N_{1} N_{2} / N_{1}^{\mathcal{N}} N_{2}^{\mathcal{N}}$ is in $\mathcal{N}$. The conclusion now follows.

Lemma 2.6 Let $L$ be a subgroup of a profinite group $G$ such that the normalizer $N_{G}(L)$ is open. Let $H$ be a normal open subgroup of $G$ contained in $N_{G}(L)$.

1. If $L$ is finite, then $\left\langle L^{G}\right\rangle$ is finite.

2. If $L$ is in $\mathcal{N}$, then so is $\left\langle(L \cap H)^{G}\right\rangle$.

3. If $L$ is finite-by- $\mathcal{N}$, then so is $\left\langle(L \cap H)^{G}\right\rangle$.

Proof Note that $L$ has only a finite number of conjugates in $G$. The first claim follows from Dicman's Lemma [7, 14.5.7]. The other ones follow from the fact that each conjugate of $L \cap H$ is a normal subgroup of $H$ and the fact that a product of finitely many normal $\mathcal{N}$-subgroups is in $\mathcal{N}$.

We recall that the Hirsch-Plotkin radical of an abstract group is defined as the product of all normal locally nilpotent subgroups. This is the maximal normal locally nilpotent subgroup. In a profinite group $G$, we have to consider closed subgroups, and the closure of the product of infinitely many normal locally nilpotent subgroups might fail to be locally nilpotent. However, if $G$ is virtually locally nilpotent, that is, if $G$ possesses an open locally nilpotent subgroup, then the product of all normal locally nilpotent subgroups of $G$ is locally nilpotent and so we can speak of the Hirsch-Plotkin radical of $G$.

Lemma 2.7 In a profinite virtually locally nilpotent group G every left Engel element is contained in the Hirsch-Plotkin radical.

Proof Let $N$ be an open normal locally nilpotent subgroup of $G$ and let $b$ be a left Engel element. Since $G / N$ is finite, by [7, 12.3.7], $b N$ belongs to the fitting subgroup $F / N$ of $G / N$. It follows that $b$ belongs to the Hirsch-Plotkin radical of $F$ and thus to the Hirsch-Plotkin radical of $G$ (see for instance [7, Exercise 12.3.7]). 


\section{Theorem 1.1}

Before proving Theorem 1.1, we need some technical results.

Lemma 3.1 Let $G=N\langle b\rangle$ be a profinite group where $N$ is an open normal finite-bypronilpotent subgroup of $G$. Assume that there exists an open normal subgroup $R$ in $G$ such that $R \leq N$ and $R\langle b\rangle$ is finite-by-pronilpotent. Then $G$ is finite-by-pronilpotent.

Proof Let $D$ be the pronilpotent residual of $N$. Since $N$ is normal in $G$, so is $D$. We can pass to the quotient $G / D$ and without loss of generality assume that $N$ is pronilpotent. Further, let $D_{1}$ be the pronilpotent residual of $\langle R, b\rangle$. This is a finite subgroup normalized by $R$. In view of Lemma 2.6, the normal closure of $D_{1}$ is finite and, factoring it out, we can assume that also $\langle R, b\rangle$ is pronilpotent.

Let $\pi=\left\{p_{1}, \ldots, p_{s}\right\}$ be the set of prime divisors of the order of the element $b N \in G / N$. Denote by $b_{i}$ a generator of the $p_{i}$-Sylow subgroup of $\langle b\rangle$ and by $P_{i}$ the $p_{i}$-Sylow subgroup of $N$. For every $i$ the subgroup $P_{i}\left\langle b_{i}\right\rangle$ is pronilpotent.

Let $T$ be the $p_{i}^{\prime}$-Hall subgroup of $N$. We remark that $\left[T, b_{i}\right]$ is finite. Indeed, $\left[R \cap T, b_{i}\right]=1$ because $\langle b, R\rangle$ is pronilpotent. Moreover $R \cap T$ is open in $T$. Thus, the centralizer of $b_{i}$ in $T$ is open. Notice that the automorphism of $T$ induced by $b_{i}$ has finite $p_{i}$-power order, since $b_{i}^{p_{i}^{n}}$ belongs to $N$ for $n$ sufficiently large and then $b_{i}^{p_{i}^{n}}$ commutes with $T$ because $N$ is pronilpotent. In view of Corollary 2.1 , it follows that $\left[T, b_{i}\right]$ is finite.

The subgroup $\left[T, b_{i}\right]$ is normalized by $T$ and is centralized by $P_{i}$ because $N$ is pronilpotent. Therefore, $\left[T, b_{i}\right]$ is normalized by $N$. Since $b$ centralizes $b_{i}$ and normalizes $T$, we conclude that $\left[T, b_{i}\right]$ is normal in $G$. So we can consider the quotient group $G /\left[T, b_{i}\right]$ and assume that $\left[T, b_{i}\right]=1$. Then, $\left\langle b_{i}, N\right\rangle=T \times\left\langle b_{i}, P_{i}\right\rangle$ and it becomes clear that $\left\langle b_{i}, N\right\rangle$ is pronilpotent.

Note that $\left\langle b_{i}, N\right\rangle$ is normal in $G$. Repeating the previous argument for each $i$, we deduce that $G$ is a product of finitely many normal pronilpotent subgroups. Hence, $G$ is pronilpotent.

Lemma 3.2 Let $G=N\langle b\rangle$ be a profinite group where $N$ is an open normal finite-by- $\mathcal{N}$ subgroup of $G$. Assume that there exists an open normal subgroup $R$ of $G$ such that $R \leq N$ and $R\langle b\rangle$ is finite-by- $\mathcal{N}$. Then $G$ is finite-by- $\mathcal{N}$.

Proof When $\mathcal{N}$ is the class of pronilpotent groups, this is precisely Lemma 3.1. Now assume that we are in the case when $\mathcal{N}$ is either the class of locally nilpotent groups or the class of strongly locally nilpotent groups.

By Lemma 3.1, $G$ is finite-by-pronilpotent. Passing to appropriate quotients without loss of generality we can assume that $N$ and $\langle R, b\rangle$ are in $\mathcal{N}$ and that $G$ is pronilpotent. Then $G / R$ is a finite nilpotent group, say of class $n$. Choose $y \in N$. Then $\left[y,{ }_{n} b\right] \leq R$. Since $R\langle b\rangle$ is locally nilpotent ( $m$-Engel, in the case where $\mathcal{N}$ is the class of strongly locally nilpotent groups), there exists an integer $m$ such that $\left[y,{ }_{n} b,,_{m} b\right]=1$. It follows that $b$ is left Engel in $G=N\langle b\rangle$ so Lemma 2.7 tells us that $b$ belongs to the Hirsch-Plotkin radical of $G$ and therefore $G$ is locally nilpotent. If $\mathcal{N}$ is the class of strongly locally nilpotent groups, $R\langle b\rangle$ is an $m$-Engel group and thus $b$ is an $(n+m)$-Engel automorphism of $N$. By Lemma 2.3 we conclude that $G$ is strongly locally nilpotent.

Lemma 3.3 Let $G=N\langle x\rangle$ be a profinite group, where $N$ is an open normal $\mathcal{N}$-subgroup of $G$. If $G$ is covered by countably many $\mathcal{N}$-subgroups, then it is finite-by- $\mathcal{N}$.

Proof Assume that $G$ is covered by countably many $\mathcal{N}$-subgroups $G_{i}$. The coset $x N$ is covered by countably many closed subsets $G_{i} \cap x N$. So by the Baire category theorem 
there exist an index $j$, an open normal subgroup $T$ of $G$ and an element $b \in x N$ such that $b(T \cap N) \leq G_{j}$. Set $R=T \cap N$ and note that $R$ is a normal open subgroup of $G$. The subgroup $\langle R, b\rangle \leq G_{j}$ is in $\mathcal{N}$. We deduce from Lemma 3.2 that $\langle N, b\rangle$ is finite-by- $\mathcal{N}$. Since $\langle N, b\rangle=\langle N, x\rangle=G$, the lemma follows.

The classical theorem of Baer says that if the $i$-th term of the upper central series of a group $G$ has finite index then $\gamma_{i+1}(G)$ is finite [7, 14.5.1]. We will require the following related result, which is due to Kurdachenko and Subbotin [6] (for our convenience we state it only for finite groups): Let $G$ be a finite group and $Z$ the last term of the upper central series of $G$. Then the order of the nilpotent residual of $G$ is bounded by a function depending only on the index $[G: Z]$.

We are now ready to complete the proof of Theorem 1.1.

Proof of Theorem 1.1 Let $G$ be a profinite group covered by countably many $\mathcal{N}$-subgroups $G_{i}$. We wish to show that $G$ is finite-by- $\mathcal{N}$. By the Baire category theorem at least one of the subgroups $G_{i}$ is open. Therefore, $G$ has a normal $\mathcal{N}$-subgroup $H$ of finite index. By Lemma 3.3, for every $x \in G$ the subgroup $\langle H, x\rangle$ is finite-by- $\mathcal{N}$. Let $D_{x}$ be the $\mathcal{N}$-residual of $\langle H, x\rangle$. Notice that by Lemma $2.6\left\langle D_{x}^{G}\right\rangle$ is finite. Since $G$ has only finitely many subgroups containing $H$, the set of subgroups $\{\langle H, x\rangle \mid x \in G\}$ is finite. Thus, $D=\prod_{x \in G}\left\langle D_{x}^{G}\right\rangle$ is a finite product of finite normal subgroups. Hence, $D$ is finite. So we can replace $G$ with the quotient group $G / D$ and assume that $\langle H, x\rangle$ is in $\mathcal{N}$ for every $x \in G$.

Let $\bar{G}$ be a finite quotient of $G$. For every $x \in G$, the image of $\langle H, x\rangle$ in $\bar{G}$ is nilpotent. Therefore, the image $\bar{H}$ of $H$ consists of right Engel elements. By [7, 12.3.7], it follows that $\bar{H}$ is contained in the last term $\bar{Z}$ of the upper central series of $\bar{G}$. Therefore, the index $[\bar{G}: \bar{Z}]$ is at most $[G: H]$. According to [6, Theorem 3.2], this implies that the order of the nilpotent residual of $\bar{G}$ is bounded by a function of $[G: H]$. As this happens in every finite quotient of $G$, the inverse limit argument shows that $G$ is finite-by-pronilpotent.

This concludes the proof when $\mathcal{N}$ is the class of pronilpotent groups.

Thus we are left with the case when $\mathcal{N}$ is the class of locally nilpotent (resp. strongly locally nilpotent) groups. We can replace $G$ by a quotient of $G$ over a finite normal subgroup and assume that $G$ is pronilpotent. Now $H$ is a normal open $\mathcal{N}$-subgroup of $G$ of finite index; we will prove by induction on the index of $H$ in $G$ that indeed $G$ is finite-by- $\mathcal{N}$. Since $G / H$ is nilpotent, we can take a non-trivial element $a H \in Z(G / H)$. By Lemma 3.3, $\langle a, H\rangle$ is finite-by- $\mathcal{N}$. Since $\langle a, H\rangle$ is normal and open in $G$, the result follows by induction.

Now to conclude the proof, it is enough to assume that $G$ is a finite-by- $\mathcal{N}$ profinite group and show that $G$ admits a covering by finitely many $\mathcal{N}$-subgroups.

Let $K$ be a normal finite subgroup such that $G / K$ is in $\mathcal{N}$ and let $N$ be an open normal subgroup of $G$ such that $N \cap K=1$. As $N$ has finite index in $G$, there are only finitely many subgroups of the form $\langle a, N\rangle$ and they cover $G$. Therefore, it is sufficient to prove that for every $a \in G$ the subgroup $\langle a, N\rangle$ is in $\mathcal{N}$. This is clear when $\mathcal{N}$ is the class of pronilpotent or locally nilpotent groups. So now we will assume that $G / K$ is $n$-Engel, and we want to prove that for every $a \in G$ the subgroup $\langle a, N\rangle$ is $n$-Engel. If $x, y \in\langle a, N\rangle$, we see that $[x, n y] \in K$ and $[x, n y] \in\langle a, N\rangle^{\prime} \leq N$. Hence $[x, n y]=1$.

\section{Theorem 1.2}

Let $G$ be a group. If $x, y \in G$, then $[x, y]=x^{-1} y^{-1} x y$ is the commutator of $x$ and $y$. The subgroup of $G$ generated by all commutators is the commutator subgroup $G^{\prime}$ of $G$. In general, elements of $G^{\prime}$ need not be commutators (see for instance [4] and references therein). Several 
recent results indicate that if the set of all commutators is covered by finitely, or countably, many subgroups with certain specific properties, then the structure of $G^{\prime}$ is similar to that of the covering subgroups. The purpose of the present section is to prove Theorem 1.2, which is a further result in this direction. The next two lemmas are similar to Lemmas 3.2 and 3.3 of [9]. For the reader's convenience, we supply the proofs.

Lemma 4.1 Suppose that the set of all commutators in a profinite group $G$ is covered by countably many $\mathcal{N}$-subgroups $G_{i}$. Then $G$ has an open normal subgroup $H$ such that $H^{\prime}$ is in $\mathcal{N}$.

Proof For each positive integer $i$ set

$$
S_{i}=\left\{(x, y) \in G \times G \mid[x, y] \in G_{i}\right\} .
$$

Note that the sets $S_{i}$ are closed in $G \times G$ and cover the whole group $G \times G$. By the Baire category theorem at least one of these sets contains a non-empty interior. Hence, there exist an open normal subgroup $H$ of $G$, elements $a, b \in G$, and an integer $j$ such that $\left[a h_{1}, b h_{2}\right] \in G_{j}$ for any choice of $h_{1}, h_{2} \in H$.

Let $K$ be the subgroup of $G$ generated by all commutators of the form $\left[a h_{1}, b h_{2}\right]$, where $h_{1}, h_{2} \in H$. Note that $K \leq G_{j}$ and that $H$ normalizes $K$. Since $G_{j}$ is in $\mathcal{N}$, so is $K$. Let $D=K \cap H$. By Lemma 2.6, $\left\langle D^{G}\right\rangle$ is in $\mathcal{N}$. Let us examine the quotient $G /\left\langle D^{G}\right\rangle$.

Suppose that $D=1$. This implies that $\left[a h_{1}, b h_{2}\right]=[a, b]$ for any $h_{1}, h_{2} \in H$. Obviously this happens if and only if $[H, a]=[H, b]=H^{\prime}=1$. Therefore $H^{\prime} \leq\left\langle D^{G}\right\rangle$ and the lemma follows.

Lemma 4.2 Suppose that the set of all commutators in a profinite group $G$ is covered by countably many $\mathcal{N}$-subgroups $G_{i}$. Then for every element $a \in G$ there exists an open normal subgroup $H_{a}$ such that $\left[H_{a}, a\right]$ is in $\mathcal{N}$.

Proof Fix an element $a \in G$ and for each positive integer $i$ let

$$
S_{i}=\left\{x \in G \mid[x, a] \in G_{i}\right\} .
$$

Note that the sets $S_{i}$ are closed in $G$ and cover the whole group $G$. By the Baire category theorem, at least one of these sets contains a non-empty interior. Hence, there exist an open normal subgroup $H$ of $G$, an element $b \in G$, and an integer $j$ such that $[h b, a] \in G_{j}$ for any $h \in H$. We have $[h b, a]=[h, a]^{b}[b, a]$. Since $[b, a] \in G_{j}$, we conclude that $[h, a]^{b} \in G_{j}$ for any $h \in H$. Therefore $[H, a] \leq G_{j}^{b^{-1}}$, which is in $\mathcal{N}$.

Lemma 4.3 If $G$ is a profinite group in which the set of commutators is covered by countably many $\mathcal{N}$-subgroups, then $G$ contains a normal subgroup $N$ such that $N$ is an open $\mathcal{N}$-subgroup of $G^{\prime}$.

Proof By Lemma $4.1 G$ possesses an open normal subgroup $H$ such that $H^{\prime}$ is in $\mathcal{N}$. Let $a_{1}, a_{2}, \ldots, a_{s}$ be elements of $G$ such that $G=\left\langle H, a_{1}, a_{2}, \ldots, a_{s}\right\rangle$. By Lemma 4.2 for every $i=1, \ldots, s$, there exists an open normal subgroup $H_{i}$ such that $\left[H_{i}, a_{i}\right]$ is in $\mathcal{N}$. Notice that $\left[H_{i}, a_{i}\right]$ is normalized by $H_{i}$, which is open and normal in $G$. Put $K_{i}=\left\langle\left[H_{i}, a_{i}\right]^{G}\right\rangle$. By Lemma 2.6, $K_{i}$ is a normal $\mathcal{N}$-subgroup for each $i$. Let $N$ be the product of $H^{\prime}$ and all subgroups $K_{i}$ for $i=1, \ldots, s$. Then, $N$ is a normal $\mathcal{N}$-subgroup of $G$ contained in $G^{\prime}$ (here we use Lemma 2.4, when $\mathcal{N}$ is the class of strongly locally nilpotent groups). Denote by $V$ the intersection of $H$ and all $H_{i}$ for $i=1, \ldots, s$. Then, $V$ is an open normal subgroup of $G$. Note that $V / N$ is centralized by $H$ and by every element $a_{i}$ for $i=1, \ldots, s$. It follows that $V / N \leq Z(G / N)$ and hence $Z(G / N)$ has finite index in $G / N$. By Schur's theorem, we conclude that $G^{\prime} / N$ is finite, as desired. 
Lemma 4.4 Let $G$ be a profinite group in which the set of commutators is covered by countably many $\mathcal{N}$-subgroups $G_{i}$. Suppose that $N$ is a normal $\mathcal{N}$-subgroup of $G$. If a is a commutator, then there exist a commutator $b \in a N$ and a normal subgroup $R$ of $G$ such that $R$ is open in $N$ and $\langle R, b\rangle$ is in $\mathcal{N}$.

Proof Let $X$ be the set of all commutators contained in the coset $a N$. Of course $X$ is nonempty. Obviously, the set $X$ is closed and therefore compact. It is clear that $X$ is covered by the (closed) subsets $X \cap G_{i}$. By the Baire category theorem, at least one of these subsets contains a non-empty interior. Hence, there exist an open normal subgroup $T$ of $G$, an element $b \in X$ and an index $j$ such that all commutators contained in $X \cap b T$ belong to $G_{j}$. Let $R=T \cap N$. Notice that for every $r \in R$, the conjugate $b^{r}$ is a commutator and $b^{r}=b[b, r] \in b R$. Since all commutators contained in $b R$ belong to $G_{j}$, it follows that $\left\langle b^{R}\right\rangle \leq G_{j}$. So $\left\langle b^{R}\right\rangle$ is in $\mathcal{N}$. We observe that $\langle b, R\rangle=\left\langle b^{R}\right\rangle R$ is a product of two normal $\mathcal{N}$-subgroups so it is in $\mathcal{N}$.

We will require the following lemma.

Lemma 4.5 [11, Lemma 3.2] Let $\pi$ be a set of primes, $G$ a finite group in which all commutators are $\pi$-elements. Then $G^{\prime}$ is a $\pi$-group.

Proposition 4.1 Let $G$ be a profinite group and let $X$ be the set of all commutators of $G$. Suppose that there exists an open subgroup $N$ of $G^{\prime}$ which is normal in $G$ such that for every $x \in X$ the subgroup $\langle N, x\rangle$ is finite-by- $\mathcal{N}$. Then $G^{\prime}$ is finite-by- $\mathcal{N}$.

Proof It will be convenient to prove the following, formally stronger, result:

If a subgroup $K$ is generated by a normal subset of $X$, the subgroup $K N$ is finite-by- $\mathcal{N}$.

Here by a normal subset we mean a subset that is invariant under any inner automorphism of $G$. Suppose that the claim is false and choose $K$ and $N$ with the property that $K N$ is not finite-by- $\mathcal{N}$ and the index $[K N: N]$ is as small as possible. Of course, whenever $H$ is a normal subgroup generated by elements from $X$ such that $N \leq H N \leq K N$ we have

(*) Either $H \leq N$ or $H N=K N$.

Indeed, suppose that none of the above holds. By minimality of the index, $H N$ is finite-by- $\mathcal{N}$. Moreover, for every $x \in X$, since the subgroup $\langle N, x\rangle$ is finite-by- $\mathcal{N}$, Lemma 3.2 implies that $\langle H N, x\rangle$ is finite-by- $\mathcal{N}$. Since $[K N: H N]<[K N: N]$, we conclude that $K N$ is finite-by- $\mathcal{N}$, a contradiction. This proves $(*)$.

If the commutator subgroup $K^{\prime}$ has the property that $K^{\prime} N<K N$, by $(*)$ we can assume that $K^{\prime} N=N$ and so $K N / N$ is abelian. Since $K N / N$ is finite, it follows that $K N$ is the product of finitely many finite-by- $\mathcal{N}$ subgroups of the form $\langle N, x\rangle$, which normalize each other. Therefore, $K N$ is finite-by- $\mathcal{N}$ by Lemma 2.5 , a contradiction.

Thus, we are left with the case where $K^{\prime} N=K N$, that is, $K N / N$ is perfect. Let $p$ be a prime divisor of $|K N / N|$ and $X_{p}$ the set of $p$-elements in $X$. The subgroup $H=\left\langle X_{p}\right\rangle$ is, of course, normal in $G$. If $H \leq N$, then by Lemma $4.5 \mathrm{KN} / N$ is a $p^{\prime}$-group, contradicting our choice of $p$. Therefore, $N \neq H N$. In view of $(*)$, it follows that $H N=K N$. Now to obtain a contradiction, it becomes sufficient to prove that $H$ is finite-by- $\mathcal{N}$ because in that case $K N=H N$ would be finite-by- $\mathcal{N}$ by Lemma 2.5 .

Let $M=H \cap N$ and $X_{H}=X \cap H$. The quotient $H / M$, being isomorphic to $H N / N=$ $K N / N$, is a finite perfect group generated by $p$-elements. Since $M$ has finite index in $H$, the set of subgroups $\left\{\langle M, x\rangle \mid x \in X_{H}\right\}$ is finite. For each $x \in X_{H}$, let $D_{x}$ be the $\mathcal{N}$-residual of $\langle M, x\rangle$. Note that each normal closure $\left\langle D_{x}{ }^{H}\right\rangle$ is finite. Therefore, $D_{0}=\prod_{x \in X_{H}}\left\langle D_{x}{ }^{H}\right\rangle$ is a finite product of finite normal subgroups and $D_{0}$ is finite. If $H \leq D_{0} N$, the result is 
immediate so we assume that $H \not \leq D_{0} N$. It follows that $H N / D_{0} N$ is perfect. Passing to the quotient group $H / D_{0}$, we can assume that the subgroup $\langle M, x\rangle$ is in $\mathcal{N}$ whenever $x \in X_{H}$.

Since $M$ is pronilpotent, $M$ is the direct product of its Sylow $p$-subgroup $P$ and its Hall $p^{\prime}$-subgroup $T$. Since the subgroups of the form $\langle M, x\rangle$ are pronilpotent, it follows that $[T, x]=1$ for every $x \in X_{p}$. Hence, $T$ is contained in the center of $H$. The quotient group $H / M Z(H)$ is a perfect finite group generated by $p$-elements. It follows from Lemma 4.5 that $H / M Z(H)$ contains some $p^{\prime}$-commutators. So the set $X_{p^{\prime}}$ of $p^{\prime}$-commutators in $X_{H}$ is nonempty. Let $L=\left\langle X_{p^{\prime}}\right\rangle$. If $L M$ were a proper subgroup of $H$, then the quotient $H / L M$ would be a perfect group with all commutators of $p$-power order. By Lemma 4.5, the commutator subgroup of $H / L M$ would have a $p$-power order as well. Thus, $H / L M$ would not be perfect, a contradiction.

Hence, $H=L M$. As before, it is sufficient to prove that $L$ is finite-by- $\mathcal{N}$. Set $M_{L}=L \cap M$, $P_{L}=L \cap P$, and $T_{L}=L \cap T$. Remark that $T_{L} \leq Z(L)$. For every $x \in X_{p^{\prime}}$, we have $\left[P_{L}, x\right]=1$ since the subgroup $\left\langle M_{L}, x\right\rangle$ is pronilpotent. Therefore, $P_{L} \leq Z(L)$ and thus $M_{L} \leq Z(L)$. It follows that $L$ is central-by-finite and so by Schur's theorem it is finite-byabelian, which gives the desired contradiction. This completes the proof.

The proof of Theorem 1.2 now becomes easy.

Proof of Theorem 1.2 If $G$ is a profinite group such that $G^{\prime}$ is finite-by- $\mathcal{N}$, then by Theorem 1.1 we have that $G^{\prime}$ is covered by finitely many $\mathcal{N}$-subgroups. So it remains to prove that if $G$ is a profinite group in which the set of all commutators is covered by countably many $\mathcal{N}$-subgroups, then $G^{\prime}$ is finite-by- $\mathcal{N}$.

Let $G$ be a profinite group in which the set of all commutators is covered by countably many $\mathcal{N}$-subgroups. By Lemma 4.3, there exists an open $\mathcal{N}$-subgroup $N$ of $G^{\prime}$ which is normal in $G$. Let $X$ be the set of all commutators in $G$ and let $a \in X$. By Lemma 4.4, there exist a commutator $b \in a N$ and a normal subgroup $R$ of $G$ such that $R$ is open in $N$ and $\langle R, b\rangle$ is in $\mathcal{N}$. Then, $R$ is open in $G^{\prime}$ and, in view of Lemma 3.2, we conclude that $\langle N, b\rangle$ is finite-by- $\mathcal{N}$. As $\langle N, b\rangle=\langle N, a\rangle$, it follows that for every commutator $a$ the group $\langle N, a\rangle$ is finite-by- $\mathcal{N}$. Now Proposition 4.1 tells us that $G^{\prime}$ is finite-by- $\mathcal{N}$, as required.

\section{References}

1. Acciarri, C., Shumyatsky, P: Coverings of commutators in profinite groups. Rend. Sem. Mat. Univ. Padova, to appear

2. Aschbacher, M.: Finite Group Theory, $2 \mathrm{~d}$ edn, vol. 10. Cambridge Studies in Advanced Mathematics. Cambridge University Press, Cambridge (2000)

3. Bastos, R., Shumyatsky, P., Tortora, A., Tota, M.: On groups admitting a word whose values are Engel. Int. J. Algebra Comput. 23, 81-89 (2013)

4. Kappe, L.C., Morse, R.: On commutators in groups. In: Proceedings of Groups St. Andrews 2005, pp. 531-558. Cambridge University Press (2007)

5. Kelley, J.L.: General Topology. Van Nostrand, New York (1955)

6. Kurdachenko, L.A., Subbotin, I.Y.: On some properties of the upper and lower central series. Southeast Asian Bull. Math. 37, 547-554 (2013)

7. Robinson, D.J.S.: A Course in the Theory of Groups, 2nd edn, vol. 80. Graduate Texts in Mathematics. Springer, New York (1996)

8. Shalev, A.: Combinatorial conditions in residually finite groups II. J. Algebra 157, 51-62 (1993)

9. Shumyatsky, P.: On profinite groups with commutators covered by nilpotent subgroups. Rev. Mat. Iberoam. 32, 1331-1339 (2016)

10. Shumyatsky, P., Sanção da Silveira, D.: On finite groups with automorphisms whose fixed points are Engel. Arch. Math. 106, 209-218 (2016)

11. Shumyatsky, P.: On groups with commutators of bounded order. Proc. Am. Math. Soc. 127, 2583-2586 (1999) 
12. Tomkinson, M.J.: Hypercentre-by-finite groups. Publ. Math. Debr. 40, 313-321 (1992)

13. Wilson, J.S.: Two-generator conditions for residually finite groups. Bull. Lond. Math. Soc. 23, 239-248 (1991)

14. Wilson, J.S., Zelmanov, E.I.: Identities for Lie algebras of pro-p groups. J. Pure Appl. Algebra 81, 103-109 (1992)

15. Zelmanov, E.: Some problems in the theory of groups and Lie algebras (Russian). Mat. Sb. 180, 159-167 (1989); translation in Math. USSR-Sb. 66, 159-168 (1990)

16. Zelmanov, E.: Solution of the restricted Burnside problem for groups of odd exponent, (Russian). Izv. Akad. Nauk SSSR Ser. Mat. 54, 42-59, 221 (1990); translation in Math. USSR-Izv. 36, 41-60 (1991)

17. Zelmanov, E.: Solution of the restricted Burnside problem for 2-groups, (Russian). Mat. Sb. 182, 568-592 (1991); translation in Math. USSR-Sb. 72, 543-565 (1992) 